\title{
A Note on Complex Algebras of Semigroups
}

\author{
Peter Jipsen \\ Chapman University, Orange CA 92866, USA \\ jipsen@chapman.edu \\ WWW home page: http://www.chapman.edu/ jipsen/
}

\begin{abstract}
The main result is that the variety generated by complex algebras of (commutative) semigroups is not finitely based. It is shown that this variety coincides with the variety generated by complex algebras of partial (commutative) semigroups. An example is given of an 8-element commutative Boolean semigroup that is not in this variety, and an analysis of all smaller Boolean semigroups shows that there is no smaller example. However, without associativity the situation is quite different: the variety generated by complex algebras of (commutative) binars is finitely based and is equal to the variety of all Boolean algebras with a (commutative) binary operator.
\end{abstract}

A binar is a set $A$ with a (total) binary operation ·, and in a partial binar this operation is allowed to be partial. We write $x \cdot y \in A$ to indicate that the product of $x$ and $y$ exists. A partial semigroup is an associative partial binar, i.e. for all $x, y, z \in A$, if $(x \cdot y) \cdot z \in A$ or $x \cdot(y \cdot z) \in A$, then both terms exist and evaluate to the same element of $A$. Similarly, a commutative partial binar is a binar such that if $x \cdot y \in A$ then $x \cdot y=y \cdot x \in A$.

Let $(\mathrm{P})(\mathrm{C}) \mathrm{Bn}$ and $(\mathrm{P})(\mathrm{C}) \mathrm{Sg}$ denote the class of all (partial) (commutative) groupoids and all (partial) (commutative) semigroups respectively. For $A \in \mathrm{PBn}$ the complex algebra of $A$ is defined as $\operatorname{Cm}(A)=\langle P(A), \cup, \emptyset, \cap, A, \backslash, \cdot\rangle$, where

$$
X \cdot Y=\{x \cdot y \mid x \in X, y \in Y \text { and } x \cdot y \text { exists }\}
$$

is the complex product of $X, Y \in \mathrm{Cm}(A)$. Algebras of the form $\mathrm{Cm}(A)$ are examples of Boolean algebras with a binary operator, i.e., algebras $\langle B, \vee, 0, \wedge, 1, \neg, \cdot\rangle$ such that $\langle B, \vee, 0, \wedge, 1, \neg\rangle$ is a Boolean algebra and $\cdot$ is a binary operation that distributes over finite (including empty) joins in each argument. A Boolean semigroup is a Boolean algebra with an associative binary operator.

For a class $\mathcal{K}$ of algebras, $\mathrm{Cm}(\mathcal{K})$ denotes the class of all complex algebras of $\mathcal{K}, \mathbf{H}(\mathcal{K})$ is the class of all homomorphic images of $\mathcal{K}$, and $\mathbf{V}(\mathcal{K})$ is the variety generated by $\mathcal{K}$, i.e., the smallest equationally defined class that contains $\mathcal{K}$.

The aim of this note is to contrast the equational theory of $\mathrm{Cm}((\mathrm{C}) \mathrm{Bn})$ with that of $\mathrm{Cm}((\mathrm{C}) \mathrm{Sg})$. It turns out that the former is finitely based while the latter is not.

Lemma $1 \mathbf{V}(\mathrm{Cm}(\mathrm{Sg}))=\mathbf{V}(\mathrm{Cm}(\mathrm{PSg}), \mathbf{V}(\mathrm{Cm}(\mathrm{CSg}))=\mathbf{V}(\mathrm{Cm}(\mathrm{PCSg}))$, $\mathbf{V}(\mathrm{Cm}(\mathrm{Bn}))=\mathbf{V}(\mathrm{Cm}(\mathrm{PBn})$ and $\mathbf{V}(\mathrm{Cm}(\mathrm{CBn}))=\mathbf{V}(\mathrm{Cm}(\mathrm{PCBn}))$. 
Proof: We prove the first result and note that the argument for the other results is identical. Since $\mathrm{Sg} \subseteq \mathrm{PSg}$, the forward inclusion is obvious.

Let $A$ be a partial semigroup, and define $A_{\infty}=\langle A \cup\{\infty\}, \cdot\rangle$ where

$$
x \cdot y=\left\{\begin{array}{l}
x y \text { if } x y \in A \\
\infty \text { otherwise. }
\end{array}\right.
$$

It is easy to check that $A_{\infty} \in \mathrm{Sg}$. Define $h: \operatorname{Cm}\left(A_{\infty}\right) \rightarrow \operatorname{Cm}(A)$ by $h(X)=$ $X \backslash\{\infty\}$. Then $(X \cdot Y) \backslash\{\infty\}=(X \backslash\{\infty\})(Y \backslash\{\infty\})$ since

$$
z=x \cdot y \text { and } z \neq \infty \quad \text { iff } \quad z=x y \text { and } x \neq \infty \text { and } y \neq \infty .
$$

Hence $h$ is a homomorphism, and it follows that $\mathrm{Cm}(\mathrm{PSg}) \subseteq \mathbf{H C m}(\mathrm{Sg})$. Therefore $\mathbf{V}(\mathrm{Cm}(\mathrm{PSg})) \subseteq \mathbf{V}(\mathrm{Cm}(\mathrm{Sg}))$.

Let Rel be the class of algebras that are isomorphic to algebras of binary relations closed under Boolean operations $(\cup, \cap, \backslash, \emptyset, T)$ and relation composition. The subclass of algebras that are commutative under composition is denoted by CRel.

Note that the top relation $T$ is always transitive. The proof below shows that the equational theory of Rel does not change even if we assume $T$ is also irreflexive (and hence a strict partial order).

Theorem $2(\mathrm{C})$ Rel is a variety, and $\mathbf{V}(\mathrm{Cm}((\mathrm{C}) \mathrm{Sg}))=(\mathrm{C})$ Rel.

Proof: The class (C)Rel is easily seen to be closed under subalgebras and products. The proof that $(\mathrm{C})$ Rel is closed under homomorphic images is similar to a proof in [2] Theorem 5.5.10 that shows cylindric-relativized set algebras are a variety (see also [6] Theorem 1.5).

Moreover, it is easy to see that $(\mathrm{C}) \operatorname{Rel} \subseteq \mathbf{V}(\mathrm{Cm}(\mathrm{P}(\mathrm{C}) \mathrm{Sg}))$ since the algebra of all subsets of a transitive relation is the complex algebra of a partial semigroup, with ordered pairs as elements, and $(w, x) \cdot(y, z)=(w, z)$ if $x=y$ (undefined otherwise).

To prove the opposite inclusion, we show that any complex algebra of a semigroup can be embedded in a member of Rel. The commutative case follows since if the semigroup is commutative then the image under this embedding will be a member of CRel.

Let $S$ be a semigroup. We would like to find a set $U$ and a collection $\left\{R_{a} \subseteq\right.$ $\left.U^{2} \mid a \in S\right\}$ of disjoint nonempty binary relations on $U$ such that $R_{a}$ 。 $R_{b}=R_{a b}$. If $S$ is a left-cancellative semigroup, we can simply take the Cayley embedding $R_{a}=\{(x, x a) \mid x \in S\}$. However, if $S$ is not cancellative then this approach does not give disjoint relations, so we take a step-by-step approach and use transfinite induction to build the $R_{a}$. A detailed discussion of this method for representing relation algebras can be found in [3] or [4]. Since our setting is somewhat different, and to avoid lengthy definitions, we take a rather informal approach here. To simplify the argument, we will arrange that all the relations are irreflexive and antisymmetric. 
Suppose we have an "approximate embedding", by which we mean a collection of disjoint irreflexive antisymmetric relations $R_{a, \kappa}$ on a set $U_{\kappa}$ such that $R_{a, \kappa} \circ R_{b, \kappa} \subseteq R_{a b, \kappa}$.

Using the well-ordering principle, we list all the pairs in $R_{a b, \kappa} \backslash\left(R_{a, \kappa} \circ R_{b, \kappa}\right)$ for all $a, b \in S$, and proceed to extend $U_{\kappa}$ and the $R_{a, \kappa}$ so as to eventually obtain $R_{a} \circ R_{b}=R_{a b}$, where $R_{a}$ is the union of all the $R_{a, \kappa}$ constructed along the way. For each $u \neq v$ with $\langle u, v\rangle \in R_{a b, \kappa} \backslash\left(R_{a, \kappa} \circ R_{b, \kappa}\right)$, choose $w \notin U_{\kappa}$ and let

$$
\begin{aligned}
& U_{\kappa+1}=U_{\kappa} \cup\{w\} \\
& R_{z}^{\prime}=\bigcup\left\{R_{x, \kappa} \circ\{\langle u, w\rangle\}: x a=z\right\} \cup \bigcup\left\{\{\langle w, v\rangle\} \circ R_{y, \kappa}: b y=z\right\} \\
& R_{a, \kappa+1}=R_{a, \kappa} \cup\{\langle u, w\rangle\} \cup R_{a}^{\prime} \\
& R_{b, \kappa+1}=R_{b, \kappa} \cup\{\langle w, v\rangle\} \cup R_{b}^{\prime} \text { and } \\
& R_{z, \kappa+1}=R_{z, \kappa} \cup R_{z}^{\prime} \text { if } z \neq a, b .
\end{aligned}
$$

For limit ordinals $\lambda$, we let $U_{\lambda}=\bigcup_{\kappa<\lambda} U_{\kappa}$ and $R_{x, \lambda}=\bigcup_{\kappa<\lambda} R_{x, \kappa}$.

It remains to check that the new relations are still an approximate embedding. By construction, they are disjoint, irreflexive and antisymmetric since $w \notin U_{\kappa}$. Checking the inclusion $R_{c, \kappa+1} \circ R_{d, \kappa+1} \subseteq R_{c d, \kappa+1}$ involves several cases, depending on whether $c, d \in\{a, b\}$. Since they are similar, we consider only the case $c, d \notin\{a, b\}$. Let $\langle p, q\rangle \in R_{c, \kappa+1} \circ R_{d, \kappa+1}$. Then there exists $r \in U_{\kappa+1}$ such that $\langle p, r\rangle \in R_{c, \kappa+1}$ and $\langle r, q\rangle \in R_{d, \kappa+1}$. If $r \in U_{\kappa}$ then the conclusion follows from the assumption that $R_{z, \kappa}$ is an approximate embedding. So we may assume $r=w$ (the unique element in $U_{\kappa+1} \backslash U_{\kappa}$ ). By construction $\langle p, u\rangle \in R_{x a, \kappa}$ for some $x$ such that $x a=c$ and $\langle v, q\rangle \in R_{b y, \kappa}$ for some $y$ such that $b y=d$. Since $\langle u, v\rangle \in R_{a b, \kappa}$ it follows that $\langle p, q\rangle \in R_{x(a b) y, \kappa}$. By associativity we have $R_{x(a b) y, \kappa} \subseteq R_{(x a)(b y), \kappa+1}=R_{c d, \kappa+1}$, as required.

Finally, to start the construction take $U_{0}$ to be a disjoint union of $S$ and $S^{\prime}=S \times\{0\}$, and for each $a \in S$ define $R_{a, 0}=\left\{\left\langle a, a^{\prime}\right\rangle\right\}$, where $a^{\prime}=\langle a, 0\rangle$.

Now the main result follows easily from the "representation theorem" that we have just established. Previously it was known from [8] that the variety generated by complex algebras of groups (i.e., the variety of group relation algebras) is not finitely based. In this case the analogous representation theorem states that every group relation algebra is representable, a result that follows directly from Cayley's theorem for groups.

Corollary $3 \mathbf{V}(\mathrm{Cm}(\mathrm{Sg}))$ and $\mathbf{V}(\mathrm{Cm}(\mathrm{CSg}))$ are not finitely based.

Proof: In [1] (Theorem 4) Andreka shows that the class Rel (called $\mathbf{R}(\cup, \cap, \mid,-$ ) in [1]) is not finitely axiomatizable, and by the preceding result $\mathrm{Rel}=\mathbf{V}(\mathrm{Cm}(\mathrm{Sg}))$. Andreka's result is proved using a sequence of finite commutative relation algebras (from [7]) such that the Boolean semigroup reducts of these algebras are not in Rel, but the ultraproduct is in CRel. It follows that CRel $=\mathbf{V}(\mathrm{Cm}(\mathrm{CSg}))$ is also not finitely axiomatizable.

In fact one can find an 8-element commutative Boolean semigroup that is not in $\mathbf{V}(\mathrm{Cm}(\mathrm{Sg}))$ : Let $A$ be the finite Boolean algebra with atoms $\{a, b, c\}$, and 
define

\begin{tabular}{c||c|c|c}
$\cdot$ & $a$ & $b$ & $c$ \\
\hline \hline$a$ & $b \vee c$ & $a \vee b$ & $a \vee c$ \\
\hline$b$ & $a \vee b$ & $a \vee c$ & $b \vee c$ \\
\hline$c$ & $a \vee c$ & $b \vee c$ & $a \vee b$
\end{tabular}

It is straight forward to check that this operation is associative. The following identity fails in this algebra but holds in $\mathbf{V}(\mathrm{Cm}(\mathrm{Sg})): s \leq t_{1} \vee t_{2} \vee t_{3} \vee t_{4} \vee t_{5}$ where

$$
\begin{aligned}
& s=x_{04} \wedge\left[\left(x_{02} \wedge\left(x_{01} \cdot x_{12}\right)\right) \cdot\left(x_{24} \wedge\left(x_{23} \cdot x_{34}\right)\right)\right] \\
& t_{1}=x_{04} \wedge\left(x_{01} \cdot \neg x_{14}\right) \\
& t_{2}=x_{01} \cdot\left[x_{14} \wedge\left(x_{12} \cdot x_{24}\right) \wedge\left(\neg y_{13} \cdot x_{34}\right)\right] \\
& t_{3}=x_{04} \wedge\left(\neg x_{03} \cdot x_{34}\right) \\
& t_{4}=\left[x_{03} \wedge\left(x_{02} \cdot x_{23}\right) \wedge\left(x_{01} \cdot \neg z_{13}\right)\right] \cdot x_{34} \\
& t_{5}=x_{01} \cdot\left[y_{13} \wedge\left(x_{12} \cdot x_{23}\right) \wedge z_{13}\right] \cdot x_{34}
\end{aligned}
$$

This identity was derived from an identity of R. Maddux for a closely related relation algebra (see e.g. [6]). To see that it holds in $\mathrm{Cm}(\mathrm{Sg})$, let $S$ be a semigroup, and assume $a_{04} \in s \subseteq S$. Then $a_{04} \in x_{04}$ and there exist $a_{02}, a_{01}, a_{12}, a_{24}, a_{23}, a_{34}$ in $S$ such that $a_{i j} \in x_{i j}$ for the given subscripts, $a_{02} \cdot a_{24}=a_{04}, a_{01} \cdot a_{12}=a_{02}$ and $a_{23} \cdot a_{34}=a_{24}$. Suppose $a_{04} \notin t_{i}$ for $i=1,2,3,4$. It remains to show that $a_{04} \in t_{5}$. Let $a_{14}=a_{12} \cdot a_{24}, a_{13}=a_{12} \cdot a_{23}$ and $a_{03}=a_{02} \cdot a_{23}$. Then $a_{04}=a_{01} \cdot a_{12} \cdot a_{24}=a_{01} \cdot a_{14} \notin x_{01} \cdot \neg x_{14}$, so $a_{14} \in x_{14}$. Moreover, since $a_{02} \notin t_{2}$, we have $a_{14} \notin \neg y_{13} \cdot x_{34}$, hence $a_{13} \in y_{13}$. Similarly $a_{03} \in x_{03}$ and $a_{13} \in z_{13}$. But now $a_{04}=a_{01} \cdot a_{13} \cdot a_{34} \in t_{5}$.

The identity fails in the algebra $A$ if one assigns $x_{02}=x_{04}=a, x_{23}=x_{24}=b$, $x_{01}=x_{12}=x_{34}=c, x_{03}=x_{14}=z_{13}=a \vee c$, and $y_{13}=a \vee b$ since in this case $s=a \wedge[a \cdot b]=a$, while $t_{1}=t_{2}=t_{3}=t_{4}=t_{5}=0$.

The following result shows that there is no smaller example.

Theorem 4 All four-element Boolean semigroups are in $\mathbf{V}(\mathrm{Cm}(\mathrm{Sg}))$.

Proof: P. Reich enumerated all four-element Boolean semigroups in [9]. There are a total of 50 (including isomorphic copies), which reduces to 28 if isomorphic copies are excluded. Of these, 6 are non-commutative with a corresponding "opposite" algebra, so only 22 need to be represented. Ten of the 22 algebras are complex algebras of partial semigroups, so by Lemma 1, they are in $\mathbf{V}(\mathrm{Cm}(\mathrm{Sg})$ ).

This leaves 12 representation problems. The operation tables for the semigroup operation of these algebras $A_{i}=\left\langle\{0<a, b<1\}, \vee, 0, \wedge, 1, \neg, \circ_{i}\right\rangle(i=$ $1, \ldots, 12)$ are listed below. Reich [9] gives finite representations for 5 of them ( $A_{1}-A_{5}$ below), leaving the remaining 7 open.

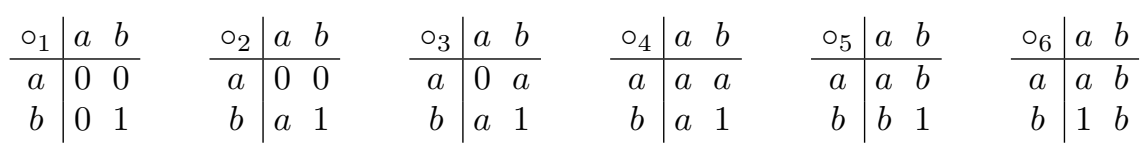




\begin{tabular}{|c|c|c|c|c|c|c|c|c|c|c|c|}
\hline $\mathrm{O}_{7}$ & $\begin{array}{ll}a & b\end{array}$ & $\circ_{8}$ & & $\circ_{9}$ & $a b$ & $\circ_{10}$ & $a b$ & $\circ_{11}$ & $\begin{array}{ll}a & b\end{array}$ & ${ }^{\circ} 12$ & $\begin{array}{ll}a & b\end{array}$ \\
\hline$a$ & $a b$ & $a$ & $\begin{array}{lll}b & 1\end{array}$ & $a$ & $\begin{array}{ll}a & a\end{array}$ & $a$ & $a 1$ & $a$ & $a 1$ & $a$ & 1 \\
\hline$b$ & 11 & $b$ & $\begin{array}{ll}1 & 1\end{array}$ & $b$ & 11 & $b$ & $1 b$ & $b$ & 11 & $b$ & 1 \\
\hline
\end{tabular}

We now indicate how to construct partial semigroups $S_{6}, \ldots, S_{12}$ and embeddings $f_{i}: A_{i} \rightarrow \mathrm{Cm}\left(S_{i}\right)$ for $i=6, \ldots, 12$. In each case it suffices to specify $f_{i}(a)$, since $f_{i}(0)=\varnothing, f_{i}(1)=S_{i}$, and $f_{i}(b)=S_{i} \backslash f_{i}(a)$.

The algebras $A_{10}, A_{11}$, and $A_{12}$ are in fact subalgebras of complex algebras of semilattices.

For $A_{10}$ take the chain $\langle\mathbb{N}, \wedge\rangle$ and define $f_{10}(a)$ to be the even numbers.

For $A_{12}$ take a countable binary tree $\left\langle B_{\infty}, \wedge\right\rangle$ (with root as the bottom element) and define $f_{12}(a)$ to be the elements of even height.

For $A_{11}$ we construct a combination of these two semilattices. Let $C_{\infty}=$ $B_{\infty} \cup B_{\infty}^{\prime}$ where $B_{\infty}^{\prime}=B_{\infty} \times\{0\}$. Each element of height $n$ in $B_{\infty}^{\prime}$ is inserted into the order of $B_{\infty}$ directly below the corresponding element of height $n$ in $B_{\infty}$ (so the root of $B_{\infty}$ becomes the root of $C_{\infty}$ ), and $f_{11}(a)=B_{\infty}$.

We note that $A_{12}$ also has a finite representation in the rectangular band $B=\left\langle\{0,1\}^{2}, *\right\rangle$, where $\langle i, j\rangle *\langle k, l\rangle=\langle i, l\rangle$ and $f_{12}(a)=\{\langle 0,0\rangle,\langle 1,1\rangle\}$.

For the remaining four algebras we are only able to give step-by-step constructions of embeddings into the complex algebra of a partial semigroup defined by a strict dense partial order. The details are similar to the proof of Theorem 2 , except that since the atoms of these algebras do not form a semigroup under . ( $=\circ_{i}$ ), the relation $R_{x y, \kappa}$ is the union of relations $R_{z, \kappa}$ where $z$ ranges over all atoms below $x \cdot y$. Hence the set of pairs $R_{z}^{\prime}=R_{z, \kappa+1} \backslash R_{z, \kappa}$ is in general not determined by a definition similar to the one given in Theorem 2 . Instead it is convenient to describe the approximate embedding relations $R_{z, \kappa}$ by a partial map $m_{\kappa}: U_{\kappa} \times U_{\kappa} \rightarrow\{a, b\}$, where $m_{\kappa}(p, q)=z$ iff $\langle p, q\rangle \in R_{z, \kappa}$. The definition of $R_{z, \kappa+1}$ is then given by a partial map $m_{\kappa+1}$. This map extends $m_{\kappa}$ and on the new pairs $\langle p, w\rangle,\langle w, q\rangle \in U_{\kappa+1} \times U_{\kappa+1}$ it is defined by the following table for the algebra $A_{6}$ :

\begin{tabular}{|c||c|cccc||c|cccc|}
\hline 1-step completion & $m_{\kappa}(p, u)$ & $a$ & $a$ & $b$ & $b$ & $m_{\kappa}(u, q)$ & $a$ & $a$ & $b$ & $b$ \\
for $A_{6}$ & $m_{\kappa}(p, v)$ & $a$ & $b$ & $a$ & $b$ & $m_{\kappa}(v, q)$ & $a$ & $b$ & $a$ & $b$ \\
\hline$m_{\kappa}(u, v)=a \leq a \circ_{6} a$ & $m_{\kappa+1}(p, w)$ & $a$ & - & $a$ & $\mathbf{b}$ & $m_{\kappa+1}(w, q)$ & $a$ & $b$ & - & - \\
$m_{\kappa}(u, v)=a \leq b \circ_{6} a$ & $m_{\kappa+1}(p, w)$ & $b$ & - & $b$ & $b$ & $m_{\kappa+1}(w, q)$ & $a$ & $b$ & - & - \\
$m_{\kappa}(u, v)=b \leq a \circ_{6} b$ & $m_{\kappa+1}(p, w)$ & - & $a$ & - & $b$ & $m_{\kappa+1}(w, q)$ & $a$ & - & $b$ & $b$ \\
$m_{\kappa}(u, v)=b \leq b \circ_{6} a$ & $m_{\kappa+1}(p, w)$ & - & $b$ & - & $b$ & $m_{\kappa+1}(w, q)$ & $a$ & - & $a$ & $b$ \\
$m_{\kappa}(u, v)=b \leq b \circ_{6} b$ & $m_{\kappa+1}(p, w)$ & - & $b$ & - & $b$ & $m_{\kappa+1}(w, q)$ & $\mathbf{a}$ & - & $b$ & $b$ \\
\hline
\end{tabular}

This table is to be interpreted as follows. Each row (after the first two) represents a choice of $x, y, z \in\{a, b\}$ and $u, v \in U_{\kappa}$ such that $m_{\kappa}(u, v)=z \leq x \circ_{6} y$ (hence $\langle u, v\rangle \in R_{z, \kappa}$ ), but $\langle u, v\rangle \notin R_{x, \kappa} \circ R_{y, \kappa}$. So one chooses $w \notin U_{\kappa}$ and defines $m_{\kappa+1}(u, w)=x$ and $m_{\kappa+1}(w, v)=y$. To complete the definition of $m_{\kappa+1}$, for each $p \in U_{\kappa} \backslash\{u\}$ the value of $m_{\kappa+1}(p, w)$ is given by the first half of the row, 
and depends on the values of $m_{\kappa}(p, u)$ and $m_{\kappa}(p, v)$ (listed in the first two rows). The table has a dash $(-)$ as entry if $m_{\kappa}(p, u) \circ_{6} m_{\kappa}(u, v) \nsupseteq m_{\kappa}(p, v)$.

The definition of $m_{\kappa+1}(w, q)$ is similar and uses the second half of the row. The entries in these rows are largely determined by the operation table for $\circ_{6}$, but in those places where a choice needed to be made, the chosen atom is listed in boldface. The appropriate choices were found by a backtrack search algorithm. It remains to check that the given definition produces relations $R_{z, \kappa+1}$ that are again an approximate embedding. This involves a tedious but straight forward case analysis. The process of refining approximate embeddings in this step-by-step way is iterated in a suitable countable sequence to ensure that $R_{z}=\bigcup_{\kappa \leq \omega} R_{z, \kappa}$ is indeed an embedding of $A_{6}$ into the complex algebra of a partial semigroup. Readers familiar with representing relation algebras by games as in [3] or [4], may note that the table above specifies a winning strategy for the existential player in such a game.

For the algebra $A_{7}$, the procedure is identical, except that the definition of $m_{\kappa+1}$ is determined by the following table:

\begin{tabular}{|c||c|cccc||c|cccc|}
\hline $\begin{array}{c}\text { 1-step completion } \\
\text { for } A_{7}\end{array}$ & $m_{\kappa}(p, u)$ & $a$ & $a$ & $b$ & $b$ & $m_{\kappa}(u, q)$ & $a$ & $a$ & $b$ & $b$ \\
$m_{\kappa}(p, v)$ & $a$ & $b$ & $a$ & $b$ & $m_{\kappa}(v, q)$ & $a$ & $b$ & $a$ & $b$ \\
\hline$m_{\kappa}(u, v)=a \leq a \circ_{7} a$ & $m_{\kappa+1}(p, w)$ & $a$ & - & $a$ & $\mathbf{b}$ & $m_{\kappa+1}(w, q)$ & $a$ & $b$ & - & - \\
$m_{\kappa}(u, v)=a \leq b \circ_{7} a$ & $m_{\kappa+1}(p, w)$ & $b$ & - & $a$ & $b$ & $m_{\kappa+1}(w, q)$ & $a$ & $b$ & - & - \\
$m_{\kappa}(u, v)=a \leq b \circ_{7} b$ & $m_{\kappa+1}(p, w)$ & $b$ & - & $b$ & $b$ & $m_{\kappa+1}(w, q)$ & $a$ & $a$ & - & - \\
$m_{\kappa}(u, v)=b \leq a \circ_{7} b$ & $m_{\kappa+1}(p, w)$ & - & $a$ & $b$ & $\mathbf{b}$ & $m_{\kappa+1}(w, q)$ & $a$ & $a$ & $b$ & $b$ \\
$m_{\kappa}(u, v)=b \leq b \circ_{7} a$ & $m_{\kappa+1}(p, w)$ & - & $b$ & $a$ & $\mathbf{b}$ & $m_{\kappa+1}(w, q)$ & $a$ & $b$ & $a$ & $b$ \\
$m_{\kappa}(u, v)=b \leq b \circ_{7} b$ & $m_{\kappa+1}(p, w)$ & - & $b$ & $b$ & $b$ & $m_{\kappa+1}(w, q)$ & $\mathbf{a}$ a & $\mathbf{b}$ & $\mathbf{b}$ \\
\hline
\end{tabular}

For $A_{8}$ one can give a similar 1-step completion table, but in this case the information in the table can be summarized by $m_{\kappa+1}(p, w)=b=m_{\kappa+1}(w, q)$ for all $p, q \in U_{\kappa} \backslash\{u, v\}$. This definition produces an approximate embedding at each step since $b \leq x \circ_{8} y, x \leq b \circ_{8} y$ and $x \leq y \circ_{8} b$ for all $x, y \in\{a, b\}$.

Finally, for $A_{9}$ the 1-step completion table is almost as easy to describe as for $A_{8}$. Here we set $m_{\kappa+1}(p, w)=b$ if $m_{\kappa}(p, u)=b=m_{\kappa}(p, v)$ and otherwise let $m_{\kappa+1}(p, w)=a=m_{\kappa+1}(w, q)$ for all $p, q \in U_{\kappa} \backslash\{u, v\}$. As before it is tedious, but not difficult to check that this definition of $m_{\kappa+1}$ again produces an approximate embedding.

It is not known whether the algebras $A_{6}, \ldots, A_{11}$ can be embedded in complex algebras of finite semigroups.

Finally we contrast the equational theory of complex algebras of semigroups with the following result adapted from [5] (Theorem 3.20).

Theorem 5 Every Boolean algebra with a binary operator can be embedded in a member of $\mathrm{Cm}(\mathrm{PBn})$. If the operator is commutative, then the algebra can be embedded in a member of $\mathrm{Cm}(\mathrm{PCBn})$.

Corollary $6 \mathrm{~V}((\mathrm{C}) \mathrm{Bn})$ ) is the variety of Boolean algebras with a (commutative) binary operator, and hence is finitely based. 


\section{References}

1. H. Andreka, Representations of distributive lattice ordered semigroups with binary relations, Algebra Universalis, 28 (1991), 12-25.

2. L. Henkin, J. D. Monk and A. Tarski, Cylindric algebras, Part II, North-Holland, Amsterdam, 1985.

3. R. Hirsch and I. Hodkinson, Step by step - building representations in algebraic logic, J. Symbolic Logic 62 (1997), 816-847.

4. R. Hirsch and I. Hodkinson, Relation algebras by games, Studies in Logic and the Foundations of Mathematics, Vol 147, Elsevier Science, North-Holland, 2002.

5. P. Jipsen, Computer aided investigations of relation algebras, dissertation, Vanderbilt University, 1992, www.chapman.edu/ jipsen/dissertation/

6. P. Jipsen and R. D. Maddux, Nonrepresentable sequential algebras, Log. J. IGPL 5 (1997), no. 4, 565-574.

7. R. D. Maddux, Nonfinite axiomatizability results for cylindric and relation algebras, J. Symbolic Logic 54(3), (1989), 951-974.

8. D. Monk, On representable relation algebras, Michigan Math. J. 11 (1964), 207-210.

9. P. Reich, Complex algebras of semigroups, dissertation, Iowa State University, 1996.

10. A. Tarski, On the calculus of relations, J. Symbolic Logic 6, (1941), 73-89. 